Made available courtesy of Cambridge University Press: http://www.cambridge.org/

***Reprinted with permission. No further reproduction is authorized without written permission from Cambridge University Press. This version of the document is not the version of record.

Figures and/or pictures may be missing from this format of the document.***

\title{
Indeterminacy is inherent in an inadequate model of evolution, not in nature
}

\section{Douglas Wahisten}

Department of Psychology, University of Waterloo, Waterioo, Ontario, Canada N2L $3 G 1$

Plotkin \& Odling-Smee's (P\&O's) critique of a "monolithic theory" of evolution espoused by prominent sociobiologists 
draws attention to the roles of learning, culture, and society in evolution. Insofar as it opposes the simple-minded genetic determinism so prevalent in this field, it shows a positive trend of thought, although it is somewhat surprising that the authors do not make reference to previous discussions of cultural transmission by Cavalli-Sforza and Feldman (1978) or by Rao et al. (1976). However, P\&O's paper has several shortcomings which make the alternative model they propose untenable.

Determinism and natural law. $\mathrm{P \& O}$ advocate that the future course of evolution is "fundamentally unpredictable" because of the indeterminacy and randomness supposedly inherent in complex interactions within and between "levels" of their model. They state boldly that "evolution is not a deterministic process."

It is instructive to refer to the opening sentence of the fifth chapter of Darwin's Origin of Species where he states: "I have hitherto sometimes spoken as if the variations-so common and multiform with organic beings under domestication, and in a lesser degree with those under nature - were due to chance. This, of course, is a wholly incorrect expression, but it serves to acknowledge plainly our ignorance of the cause of each particular variation."

The problem with a proclamation of randomness and unpredictability is that it may merely reify the theorist's own lack of knowledge and understanding, thereby discouraging further efforts to investigate and analyse phenomena. $\mathrm{P} \& \mathrm{O}$ are quite right in their opinion that living systems entail a plethora of exceedingly complex interactions that can never be understood by simplistic sociobiology, but I think they are wrong in their despair about prospects of understanding this web of interaetions.

Furthermore, the present difficulty of predicting the future should not be made to weigh so heavily on only one aspect of the problem - the evolution of organic species. We cannot even predict what the inorganic environment will be like millenia from now, although the properties of this environment, of course, depend to some extent on developments in the realm of life. At any rate, without an accurate estimation of the conditions of life, it is impossible to predict the responses of living beings to changed conditions.

The complexity of the system shows the need for a great deal more investigation of nature and of real interactions before we retire to the armchair and pass judgment on nature.

Adaptation and natural selection. $\mathrm{P} \& \mathrm{O}$ assert that natural selection is "one of nature's universals," which evaluates "culturally derived adaptations in the same way as any other adaptation"; this implies that selection is the basis for all evolutionary progress.

There is no doubt that there can be competitive struggle between cultures that may even lead to the extinction of certain features of a culture. However, adaptation or innovation and cultural transmission can also take place without selection. Because of the rapidity of cultural transmission throughout a population within an individual's lifetime, and because cultural innovations come into being as solutions for widespread problems (e.g. disease) in nonrandom ways, it is possible for a new adaptation to be universally adopted in short order. This does not free a species from the reality of natural selection, but it does show that adaptation and selection are two separate and distinct sources of evolutionary change. Through cultural adaptation and transmission, progress is possible without the need for part of the population to fail. The conditions of living for the whole of society can be elevated.

The fundamental tautology. When P\&O tell us that "one can never escape from natural selection," they seem to be using selection in its tautological sense: the most fit will propagate best; those that propagate best shall be called the most fit. Darwin rose above this mundane level by proposing
that natural selection, which was obvious to almost everyone was in fact a creative force, which could lead to the develop ment of a new species - something qualitatively differen from its ancestors.

$\mathrm{P} \& \mathrm{O}$ do not inform us about the fundamental problem of evolution: speciation. In some places they try to account for why a species is well-adapted to the conditions of life here and now, and why certain historical changes may occu within a species. But on a central problem of evolutionary theory today, the gradual or abrupt transition to a new species, their model is silent.

$P \& O$ 's speculations about genetic determination of lean. ing ability and the "capacity for culture" are topics of current debate relating to attributes of human races and social classes and in the present target article these are topies presented in simple terms that could easily be misunderstood or misused

The gene-learning alchotomy. Like so many others in this field (e.g., Lorenz) $\mathrm{P} \& \mathrm{O}$ adhere to the dogma that there are only two means of information acquisition and transmission genes and learning. This reveals a rather narrow perspective on heredity. Consider just three of the many other mecha nisms: Cytoplasmic inheritance (Grun 1976) involves trans. mission of nonnuclear organelles and structural details such as mitochondria, endoplasmic reticulum, Golgi apparatus, ell membrane, etc. Certain of these contain their own DNA but many do not. The key thing here is that cytoplasmic struc tures are not organized de novo by gene products acting on a disorganized pool of central intermediates. On the contrary, new structures are built using existing structures as templates (Lehninger 1967). The fertilized egg contains a diverse array of structures in a well-organized cytoplasm, and these nongenetic components of heredity are reproduced and trans. mitted from old cells to new.

The position of a structure in the cell can be transmitted to new cells. This is clearly evident in protozoa, where microsur gical modifications of the cilia in the cell surface are perpetur ated for many generations. The importance of such "posit tional information" is now being recognized in multicellular organisms as well (see Subtelny \& Konigsberg 1979). [See also Corballis \& Morgan: "The Biological Basis of Human Later ality" $B B S 2$ (2) 1978.]

In mammals, inheritance may also entail transmission of viruses, antibodies or various chemicals directly from mother to offspring via placental transfer or the milk; even transmis sion of an acquired immunity via the father has been claimed (Steele 1979).

Thus $P \& O$ 's assertion that animals "cannot transmit any of their noncultural adaptations nongenetically" is simply not valid. This omission is especially serious because nongenetic mechanisms of inheritance appear to be more plastic than chromosomal DNA-based mechanisms; they are downright Lamarckian in some instances.

Culture in a nutshell. $\mathrm{P} \& \mathrm{O}$ argue the interesting point that natural selection makes no distinction "between a cultural and a noncultural adaptation within the same animal"; this is similar to saying that selection acts on phenotypes, not genotypes. This notion raises a very big question about the meaning of culture. The authors consider culture to be founded upon associative learning ability, but this scheme leaves out a host of nongenetic, nonlearning aspects of life that appear to function almost the same way that culture, narrowly defined, does.

Consider an oak tree laden with acorns. The fact that the tree has reached reproductive maturity proves that its envi ronment nurtures that particular species of oak. By dropping its acorns onto fertile ground, the parent transmits one aspect of its environment to its offspring. And what about the nutrient-rich endosperm and the seed coat of the nut itself? They are separate from the embryo proper, yet they are 
transmitted from parent to offspring

Between nuclear genes and associative learning there is a vast profusion of phenomena in plants and animals alike, and ny theory, such as the one diagrammed in P\&O's Figure 2 , which totally neglects these is not a yalid reflection of nature bit is, rather, a product of imagination inspired by narrowly circumscribed facts about nature. Having left so much out of their analysis, it is no wonder that $\mathrm{P} \& \mathrm{O}$ cannot even begin to inake accurate predictions about the future course of evolution.

\section{A defense of monolithic sociobiology and genetic mysticism}

\section{George C. Williams}

obeartment of Ecology and Evolution, State University of New York, Stony Brook, N.Y, 11794

Plotkin \& Odling-Smee (P\&O) give a nicely reasoned account of the way natural selection, by adjusting gene frequencies when it acts on phenotypes, has its lasting effects. Selection is always between organisms, never within, and it is always on ohenotypes transcribed from genotypes, with no possibility of communication from phenotype to gene. However complex the events of $\mathrm{P} \& \mathrm{O}$ 's levels 2 and 3 might be, these levels have evolutionary significance only to the extent that they affect the gene-frequency arrays of level 1 . I am a bit surprised at the implication that sociobiologists, radical or otherwise, thould see anything controversial in recognizing that evolution can provide organisms ". . with some capacity for autonomous information gain and storage." Organs that collect and use information are no less biological than those that collect and use food. I suspect that the distinction between levels 2 and 3 is a bit arbitrary, and that more levels might be recognized, but this would not affect their reasoning or the validity of the monolithic theory for phenotypic features at these levels.

There is nothing arbitrary about level 4. P\&O show convinicingly that there is a novel process here and that level 4 requires some special attention not needed at lower levels. The novel process is cultural inheritance, which may be considered Lamarckian only in the special sense that inherted features are not coded in the genes, rather than in the usual sense that phenotypic features are being transcribed onto the germ plasm. I can find no fault with the statement of conditions necessary for cultural inheritance. Also, I accept most of their comparisons between level- 1 and level- 4 modes of inheritance, but I believe that P\&O are led astray by a talure to appreciate some of the similarities.

Cultural traits, memes as Dawkins's (1976) cited book terms them, are subject to natural selection - not because they affect the fitness of organisms - but because they are replica. tors (like genes) and have a mechanism of inheritance. The matural selection of memes is subject to the same rules and limitations as the natural selection of genes. A gene may be fovorably selected without having developmental effects (by regregation, distortion, and other processes) or in spite of What might be considered unfortunate effects. Sexual competition, for example, may select for genes that reduce both individual viability and efficiency of resource utilization by The group. Likewise, cultural traits may be favorably selected lespite unfortunate consequences for biological fitness, perceived well-being, or group survival. Astrology, celibate Presthoods, potlatch ceremonies, and the chewing of bubble tun are a few examples.

For a cultural feature to prevail, it is necessary and sufficient for it to be effective at replicating itself in the environthent provided by society. It is not necessary that it be adaptive for that society or any of its members. Note that $P \& O$ speak of cultural adaptive traits but nowhere recognize that cultural evolution can favor traits that are biologically maladaptive. They themselves clearly state the reason for this: the nongenetic mode of inheritance of cultural traits makes them partly independent of the fitness of their carriers. There may be societies in which Russian roulette would spread more readily than sound dietary innovations. $P \& O$ fail to recognize that the theory of evolution by natural selection is applicable wherever replicators arise, and that biological evolution based on those replicators called genes is merely a special case of the more general theory. The flaw in monolithic evolution, as they describe it, is that it is not monolithic enough.

I fail to understand $P \& O$ 's rejection of purpose at the level of the gene. The prevailing allele at a given locus owes its prevalence to an adaptive molecular structure. That structure is one of an astronomical number of possibilities, and it must be one of the best among all that have ever arisen at that locus, at getting itself replicated. This high level of functional effectiveness is the same sort of consideration that $P \& O$ use to argue that pink feet can have a teleonomic purpose. In fact, the argument is more reasonable for genes than for developed characters. All extant pink feet will soon be gone, but a gene with a role in the development of pink feet can grow ever more numerous through evolutionary time. Natural selection reliably maximizes properties like adaptation, and fitness, and selfishness only at the level of the replicators themselves (genes and memes). Such properties emerge at phenotypic and societal levels only with qualifications. The genetic mysticism that $P \& O$ condemn is preferable to the phenotypic mysticism that they condone.

Any comprehensive theory has limitations, and it is possible to identify a limitation and label it a flaw, Sociobiology is an attempt to explain social behaviors as adaptations to the environment, especially the social enviromment. It does not address questions of behavioral ontogeny except in the limited sense of recognizing that ontogenetic mechanisms, such as learning capacities, are developed characters that must have ultimate evolutionary explanations. $\mathrm{P} \& \mathrm{O}$ are right that the monolithic theory is of little help in estatblishing links among genes, development, learning and socioculture. Their quotation from Rosenblatt (1976) is also valid; we need a convincing way of relating these different levels of behavioral organization, and this need is not met by sociobiology. My guess is that their multilevel theory is a bit too abstract a level to constitute an effective beginning for a theory of behavioral ontogeny. If and when we get a detailed scientific picture of the development of social behavior in human and other cultural animals, I would expect sociobiology to have a role in explaining phylogenetic variation in the details of that picture.

\section{Authors' Response}

\section{Possible mechanisms for a multiple-level model of evolution}

\section{H.C. Plotkin and F.J. Odling-Smee}

'Department of Psychology, University College London, London WC IE 6BT. England and 'Departments of Biology and Psychology, Brunel University, Uxbridge UBA $3 P H_{1}$ England

Many of the commentaries cohere around two major points of criticism. The first is that we have omitted discussion of the mechanisms that are assumed to operate at levels 2, 3, and 4. Campbell, Cloak, Dews- 